\title{
Polyhedral embeddings of snarks with arbitrary nonorientable genera
}

\author{
Wenzhong Liu* \\ Department of Mathematics \\ Nanjing University of Aeronautics and Astronautics \\ 210016 Nanjing, P.R. China \\ wzhliu7502@nuaa.edu.cn \\ Yichao Chen $^{\dagger}$ \\ College of Mathematics and Econometrics \\ Hunan University \\ 410082 Changsha, P. R. China \\ ycchen@hnu.edu.cn
}

Submitted: Nov 26, 2011; Accepted: Jul 10, 2012; Published: Aug 9, 2012

Mathematics Subject Classifications: 05C10, 05C15

\begin{abstract}
Mohar and Vodopivec [Combinatorics, Probability and Computing (2006) 15, 877-893] proved that for every integer $k(k \geqslant 1$ and $k \neq 2)$, there exists a snark which polyhedrally embeds in $\mathbb{N}_{k}$ and presented the problem: Is there a snark that has a polyhedral embedding in the Klein bottle? In the paper, we give a positive solution of the problem and strengthen Mohar and Vodopivec's result. We prove that for every integer $k(k \geqslant 2)$, there exists an infinite family of snarks with nonorientable genus $k$ which polyhedrally embed in $\mathbb{N}_{k}$. Furthermore, for every integer $k(k>0)$, there exists a snark with nonorientable genus $k$ which polyhedrally embeds in $\mathbb{N}_{k}$.
\end{abstract}

Keywords: polyhedral embedding; snark; nonorientable surface; nonorientable genus; Euler genus

\section{Introduction}

During a conference in 1968, Grübaum [5] conjectured that each cubic graph with a polyhedral embedding in an orientable surface is 3-edge-colourable. A positive solution

${ }^{*}$ The first author is the corresponding author, supported by "The Fundamental Research Funds for the Central Universities", No. NS2012143

†The work of the second author was partially supported by NNSFC under Grant No. 10901048 
of this conjecture would generalize the dual form of the Four-Color-Theorem to every orientable surface. The conjecture holds for the sphere from the results of Tait [16] and Apple and Haken [1]. In [9], Kochol disproved the conjecture by showing that there exist infinitely many snarks with polyhedral embeddings in $\mathbb{S}_{k}(k \geqslant 5)$. The smallest of the counterexamples found by Kochol is a snark of order 74. With the aid of computer, Mohar and Vodopivec [14] proved that for every cubic graph with fewer than 30 vertices, the conjecture holds true. Furthermore, they proved that for every integer $k(k \geqslant 1$ and $k \neq 2$ ), there exists a snark which polyhedrally embeds in $\mathbb{N}_{k}$, and proposed the following problem:

Problem 1. (Problem 5.3 of [14]) Is there a snark that has a polyhedral embedding in the Klein bottle?

In the paper, we give a positive solution of Problem 1 and strengthen Mohar and Vodopivec's result. Actually, we prove that for every integer $k(k \geqslant 2)$, there exists an infinite family of snarks with nonorientable genus $k$ which polyhedrally embed in $\mathbb{N}_{k}$. Furthermore, for every integer $k(k>0)$, there exists a snark with nonorientable genus $k$ which polyhedrally embeds in $\mathbb{N}_{k}$.

\section{Preliminary}

All graphs considered in this paper are connected. For some terminologies without description here, we may refer the reader to $[4,6,13]$.

A surface is a compact closed 2-dimensional manifold without boundary. In topology, surfaces are classified into the orientable surface $\mathbb{S}_{m}$, with $m$ handles $(m \geqslant 0)$ and the nonorientable surface $\mathbb{N}_{k}$, with $k$ crosscaps $(k>0)$. A graph embedding into a surface means a cellular embedding, so that every face of the embedding is an open topological disk. The orientable genus $\gamma(G)$ of a graph $G$ is the smallest integer $k$ such that $G$ cellularly embeds into $\mathbb{S}_{k}$. Similarly, the nonorientable genus $\widetilde{\gamma}(G)$ of a graph $G$ is the smallest integer $k$ such that $G$ cellularly embeds into $\mathbb{N}_{k}$. The Euler genus $\bar{\gamma}(G)$ of a graph $G$ is defined as $\min \{2 \gamma(G), \widetilde{\gamma}(G)\}$. Also note that $\bar{\gamma}(G)=\min \{2-\chi(\mathbb{S}) \mid$ G cellularly embeds into a surface $\mathbb{S}\}$, where $\chi(\mathbb{S})$ denotes the Euler characteristic of a surface $\mathbb{S}$. By the well-known formula $\widetilde{\gamma}(G) \leqslant 2 \gamma(G)+1$, either $\widetilde{\gamma}(G)=\bar{\gamma}(G)$ or $\widetilde{\gamma}(G)=\bar{\gamma}(G)+1$.

A graph $G$ is called a k-amalgamation of two graphs $G_{1}$ and $G_{2}$, denoted by $G=$ $G_{1} \bigcup_{k} G_{2}$, if $G=G_{1} \cup G_{2}$ and $G_{1} \bigcap G_{2}$ is a set of $k$ vertices. In [2], Archdeacon proved the following theorem:

Lemma 2. ([2], Theorem 1.1)

$$
2-2 k \leqslant \bar{\gamma}\left(G_{1}\right)+\bar{\gamma}\left(G_{2}\right)-\bar{\gamma}\left(G_{1} \bigcup_{k} G_{2}\right) \leqslant k^{2}-4
$$

An embedding of a graph $G$ is called polyhedral if all facial walks are cycles, and any two of them are either disjoint, intersect in one vertex, or intersect in one edge. If $G$ is 
a cubic graph, then any two facial walks are either disjoint or intersect in precisely one edge.

A snark is a cyclically 4-edge-connected cubic graph of girth at least 5 with no 3-edgecoloring. A graph is called a 4-snark if it dose not admit a nowhere-zero $\mathbb{Z}_{2} \times \mathbb{Z}_{2}$-flow. It is well known that a nowhere-zero $\mathbb{Z}_{2} \times \mathbb{Z}_{2}$-flow in a cubic graph $G$ corresponds to a 3edge-coloring of $G$. Thus snarks form a proper subclass of 4 -snarks. Kochol [8] introduced a general method to construct a 4-snark. It is based on the following two steps.

Suppose $v$ is a vertex of a graph $G$ and a graph $G^{\prime}$ is obtained from $G$ by the following process. Replace $v$ by a graph $H_{v}$ so that each edge $e$ of $G$ having one end $v$ now has one end from $H_{v}$. If $e$ is a loop incident with $v$, then both ends of $e$ will now be from $H_{v}$. We call $G^{\prime}$ a vertex superposition of $G$.

Suppose $e$ is an edge of $G$ with ends $u$ and $v$ and a graph $G^{\prime}$ is constructed from $G$ as follows: replace $e$ by a graph $H_{e}$ having at least two vertices. In other words, we delete $e$ from $G$, pick two distinct vertices $u^{\prime}, v^{\prime}$ of $H_{e}$, and identify $u^{\prime}$ with $u$ and $v^{\prime}$ with $v$. We call $G^{\prime}$ an edge superposition of $G$. If $H_{e}$ is a 4-snark, then $G^{\prime}$ is called a 4-strong edge superposition of $G$.

A graph $G^{\prime}$ is called a (4-strong) superposition of a graph $G$ if $G^{\prime}$ is obtained from $G$ by some vertex and (4-strong) edge superpositions. Kochol [8] proved the following lemma:

Lemma 3. ([8], Lemma 4.4) Let $G$ be a 4-strong superposition of a 4-snark, then $G$ is a 4-snark.

\section{Main theorem}

The Petersen graph $P$ is the smallest snark and has a polyhedral embedding in the projective plane, indicated in part $(b)$ of Figure 1.

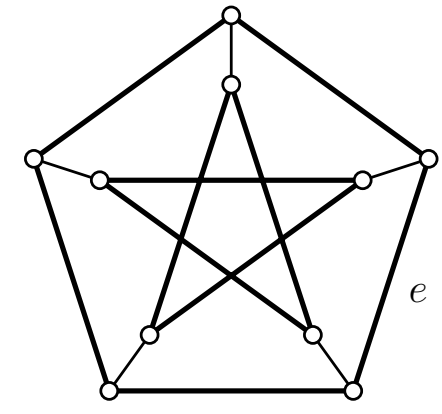

(a)

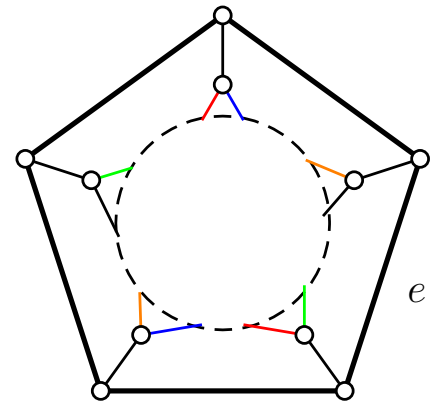

(b)

Figure 1: $(a)$ the Petersen graph $P$ and $(b)$ the Petersen graph $P$ polyhedrally embeds in the projective plane 


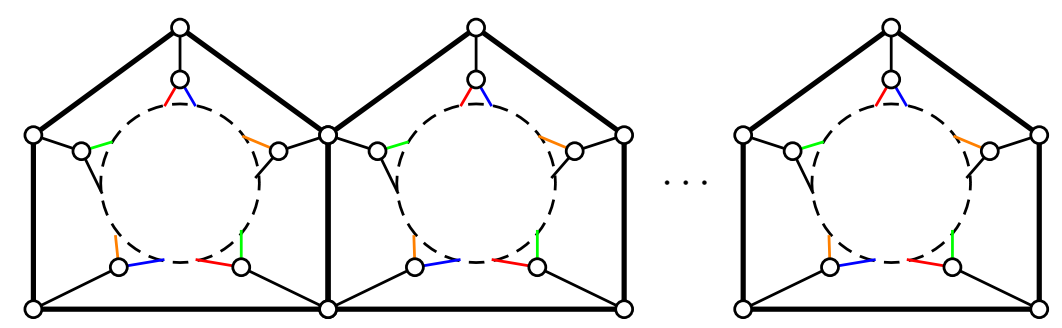

Figure 2: The graph $G_{8 k+2}$ resulting from $k$ copies of the Petersen graph polyhedrally embeds in $\mathbb{N}_{k}$

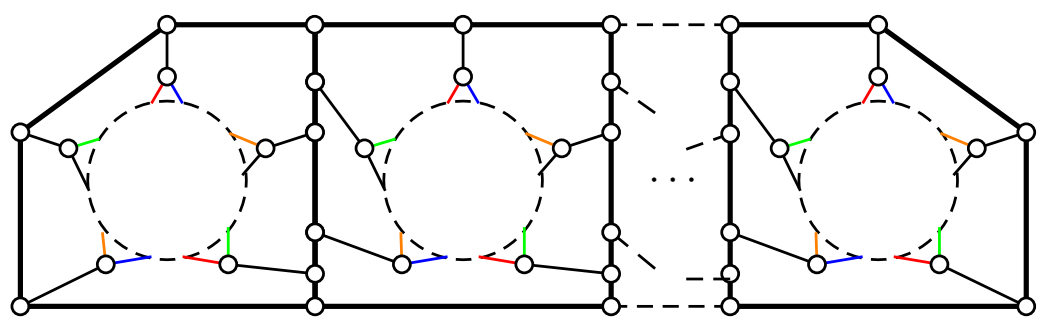

Figure 3: The snark $S_{12 k-2}$ polyhedrally embeds in $\mathbb{N}_{k}$

Theorem 4. For every integer $k(k \geqslant 2)$, there exists an infinite family of snarks with nonorientable genus $k$ which polyhedrally embed in $\mathbb{N}_{k}$.

Proof. The proof comprises the following two parts:

$(a)$. For every integer $k(k \geqslant 2)$, we construct an infinite family of snarks with polyhedral embeddings in $\mathbb{N}_{k}$;

$(b)$. For every integer $k(k \geqslant 2)$, we prove that the snarks which are constructed in part $(a)$ have nonorientable genus $k$.

The Petersen graph $P$ has a polyhedral embedding in $\mathbb{N}_{1}$ as shown in part $(b)$ of Figure 1. By applying 4-strong edge superposition $(k-1)$ times so that the edge $e$ is recurrently replaced by the copy of $P$, we get the graph $G_{8 k+2}$ of order $8 k+2$, shown in Figure 2. Replacing all vertices of degree 5 in $G_{8 k+2}$ by the paths of order 3 , we obtain the graph $S_{12 k-2}$ of order $12 k-2$ (see Figure 3). By Lemma 3, $S_{12 k-2}$ is a 4 -snark. Then $S_{12 k-2}$ has no 3-edge-coloring. Since $S_{12 k-2}$ is a cubic graph, it has a 4-edge-coloring by Vizing theorem. Because the Petersen graph is cyclically 4-edge-connected and has girth $5, S_{12 k-2}$ is cyclically 4 -edge-connected and has girth 5 . So $S_{12 k-2}$ is a snark. It has a polyhedral embedding in $\mathbb{N}_{k}$, indicated in Figure 3.

In $G_{8 k+2}$, replace all vertices of degree 5 by paths of order 3 or by graphs $C_{i, 5}(i \geqslant 1)$, drawn in Figure 4. Dashed lines indicate the edges incident with a vertex of degree 5 in $G_{8 k+2}$. The resulting graphs are vertex superpositions of $G_{8 k+2}$ and are snarks by Lemma 3. Thus we construct an infinite family of snarks with polyhedral embeddings in $\mathbb{N}_{k}$. 

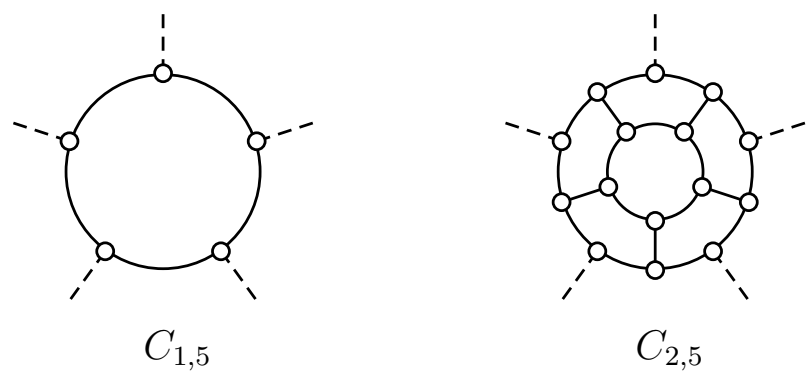

Figure 4: The graph $C_{i, 5}(i=1,2)$

Now, we prove part $(b)$. Let $k=2$ in Lemma 2 , we get

$$
\bar{\gamma}\left(G_{1} \bigcup_{2} G_{2}\right) \geqslant \bar{\gamma}\left(G_{1}\right)+\bar{\gamma}\left(G_{2}\right) .
$$

Since the Petersen graph $P$ has orientable genus 1 and nonorientable genus $1, \bar{\gamma}(P)=1$ from the Euler genus definition. Recurrently computing $\bar{\gamma}\left(G_{8 i+2}\right)$ from $i=2$ to $i=k$ (see Figure 2), we can deduce $\bar{\gamma}\left(G_{8 k+2}\right) \geqslant k$ according to the inequality (1). For every graph $G, \widetilde{\gamma}(G)=\bar{\gamma}(G)$ or $\widetilde{\gamma}(G)=\bar{\gamma}(G)+1$. Thus we get $\widetilde{\gamma}\left(G_{8 k+2}\right) \geqslant k$. Since $G_{8 k+2}$ has an embedding in $\mathbb{N}_{k}$ (see Figure 2), $\widetilde{\gamma}\left(G_{8 k+2}\right)=k$ is deduced.

When replacing all vertices of degree 5 in $G_{8 k+2}$ by paths of order 3 or by graphs $C_{i, 5}$ $(i \geqslant 1)$, the resulting graphs are snarks according to the previous argument. It is clear that the obtained snarks have nonorientable genus $k$ because $\widetilde{\gamma}\left(G_{8 k+2}\right)=k$ and paths of order 3 or graphs $C_{i, 5}(i \geqslant 1)$ are all planar graphs.

Corollary 5. For every integer $k(k>0)$, there exists a snark with nonorientable genus $k$ which polyhedrally embeds in $\mathbb{N}_{k}$.

Proof. This is directly deduced from Theorem 4 and the fact that the Petersen graph has a polyhedral embedding in the projective plane.

\section{Acknowledgements}

The authors are grateful to the referees for their useful suggestions. Thanks are given to Professor Tommy Jensen for his patience and detail comments on a former version of the paper.

\section{References}

[1] K. Appel and W. Haken, Every planar map is four colorable, Contemp. Math. 98 Amer. Math. Soc., Providence RI, 1989.

[2] D. Archdeacon, The nonorientable genus is additive, J. Graph Theory 10 (3) (1986) 363-383. 
[3] S. Belcastro and J. Kaminski, Families of dot-product snarks on orientable surfaces of low genus, Graphs Combin. 23 (2007) 229-240.

[4] R. Diestel, Graph Theory, 3rd edn, Springer, Berlin, 2005.

[5] B. Grünbaum, Conjecture 6, In Recent Progress in Combinatorics (W. T. Tutte, ed.), Academic Press, New York, 1969, p. 343.

[6] J. L. Gross and T. W. Tucker, Topological Graph Theory, Dover, 2001 (original edn. Wiley, 1987).

[7] R. Isaacs, Infinite families of nontrivial trivalent graphs which are not Tait colorable, Amer. Math. Monthly 82 (1975) 221-239.

[8] M. Kochol, Superposition and constructions of graphs without nowhere-zero k-flows, Eur. J. Combin. 23 (2002) 281-306.

[9] M. Kochol, Polyhedral embeddings of snarks in orientable surfaces, Proc. Amer. Math. Soc. 137 (5) (2009) 1613-1619.

[10] W. Z. Liu, Y. P. Liu and Y. Xu, A census of boundary cubic rooted planar maps, Discrete Appl. Math. 155 (2007) 1678-1688.

[11] W. Z. Liu and Y. P. Liu, The number of rooted essential maps on surfaces, Acta Math. Sin. 8(24)(2008) 1303-1312.

[12] G. Miller, An additivity theorem for the genus of a graph, J. Combin. Theory Ser. B 43 (1) (1987) 25-47.

[13] B. Mohar and C. Thomassen, Graphs on Surfaces, Johns Hopkins University Press, Baltimore, London, 2001.

[14] B. Mohar and A. Vodopivec, On polyhedral embeddings of cubic graphs, Combin. Probab. Comput. 15 (2006) 877-893.

[15] B. Mohar and A. Vodopivec, The genus of Petersen powers, J. Graph Theory 67 (2011) 1-8.

[16] P. G. Tait, Remarks on the colouring of maps, Proc. Soc. Edinburgh 10 (1880) 729.

[17] F. C. Tinsley and J. J. Watkins, A study of snark embeddings, Graphs and Applications (Boulder, CO, 1982), Wiley-Interscience Publication, New York, 1985, 317-332. 